\title{
Exploring User Requirements for Non-visual Mobile Navigation Systems
}

\author{
Charlotte Magnusson ${ }^{1}$, Kirsten Rassmus-Gröhn ${ }^{1}, \operatorname{Konrad}_{\text {Tollmar }}{ }^{1,2}$, \\ and Hanna Stigmar ${ }^{3}$ \\ ${ }^{1}$ Certec, Department of Design Sciences, Lund University, \\ Box 118, 22100 Lund, Sweden \\ ${ }^{2}$ Mobile Life, DSV, Stockholm University, Forum 100 \\ SE-16440 Kista, Sweden \\ ${ }^{3}$ GIS-centrum, Lund University, Sölvegatan 12, 22362 LUND \\ kirre@certec.1th.se, charlotte.magnusson@certec.lth.se, \\ konrad.tollmar@design.lth.se, hanna.stigmar@lantm.lth.se
}

\begin{abstract}
This paper describes an explorative user study of how two different user groups experience current, as well as envisioned new mobile navigation systems. Two groups have been the primary target in this study; a group of elderly people and a group of relatively young university students that were visually impaired. The study consisted of three parts: a focus group/test, a diary study and a design workshop where the users envisioned new kinds of interaction with mobile navigation systems by building and demonstrating low-fi prototypes. Information about user requirements for these types of applications is obtained and we observe features in the study design which are relevant for a wider range of mobile services.
\end{abstract}

Keywords: Mobile, Navigation, Non-visual, Digital Maps.

\section{Introduction}

The present study, which is part of the HaptiMap project (FP7-ICT-224675. Cofunded by the European Commission and also supported by VINNOVA, Sweden), investigates user requirements for the design of mainstream mobile navigational devices and mobile maps, with a particular focus on the non-visual modalities. The project targets several user groups: sighted, elderly and visually impaired persons.

A growing number of mobile applications (to a large degree map-based navigation systems) make heavy use of visual information while implicitly targeting usage situations where the visual channel is needed for other things than looking at a device [1]. Despite this interfaces used in mobile devices are usually based on traditional visual map design [2] and more flexible and usable interfaces for geospatial interaction have not yet been properly explored.

\section{Exploring Non-visual Mobile Navigation Systems}

This study targets several user groups and the design space to be investigated is complex. Furthermore, we do not look at assistive devices for a selected group, but are 
interested in improving the design of mainstream products and services. We are in the process of investigating the user requirements relevant to this particular project, and the present article reports an early explorative study involving both elderly and visually impaired users.

In the design of our study were inspired by [3 - 6] and combined a focus group discussion, a contextual test/interview, a diary study and a user workshop where users design low-fi versions of potential services. Often this type of sequence is described as separate studies - but in our study design we decided to link the different parts together. Thus, the two initial activities were important on their own, but also expected to provide the necessary background for the user workshop.

The study consisted of three parts. The first was a discussion/focus group meeting where the groups also tested different navigational systems informally in a scenario walk. The second part consisted of a diary study performed over a week where the participant recorded his or her experiences of everyday routines when traveling. The final and third part of this study was the design workshop in which each group envisioned new kinds of interaction with mobile navigation systems by building and demonstrating low-fi prototypes and demonstrated these in a simulation walk [Fig 1].
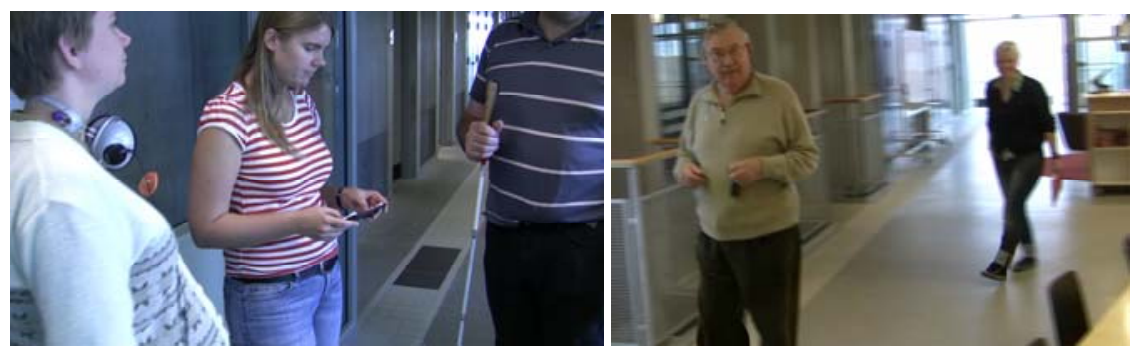

Fig. 1. Demonstrating low-fi prototypes in a simulation walk

The study involved a group of eight persons; five elderly people between 67-78 (2 male / 3 female) with a general reduction in vision capabilities and a group of three relatively young university students that are visually impaired ( 1 male / 2 female).

Almost everyone in the group claimed that they performed daily trips, mostly by public transportation. Leisure was the most common reason to go for a trip (3-5 times/week) followed by shopping (3-5 times/week), and work (3-5 times/week, and of course in this group most participants do not work). Everyone in the group owns a PC and cell phone but only two persons own a GPS. They all use Internet daily but never mobile services and almost never navigation systems. Travel planning is mostly done via Internet and printed maps are brought on the trip. When going on a trip they all bring there their cell phone, and sometimes maps and time tables. But company is also needed in some cases since they can't always operate on their own.

\section{Summary of Results and Discussion}

Both our user groups turned out to have many requirements in common. Both groups pointed out the importance of landmarks and context information such as houses, 
house numbers and other things in the environment. Both groups were in general interested in hands-free and eyes-free solutions, although one of the visually impaired users wanted a device that had a fairly large screen which could act as a device that made it possible to see things further away. Both groups also liked speech information although it was seen as important that this should not be too disturbing. Confirmation that you are on the right track was also important for both groups as well as position accuracy. Both groups were also concerned about rerouting - if you indeed made a wrong turning you would prefer to get that information to being rerouted. Information about your orientation in the environment is another common requirement as (of course) correct and updated map information. The main difference between the groups was in fact the attitude to multifunctional devices - the elderly group wanted a simple device with few functions, while the younger visually impaired users were more concerned with the number of devices and preferred one device that could do everything. The diversity found (particularly within the group of visually impaired users) still highlights the importance of being able to tailor the interaction and the information to the needs of the particular user. As can be expected these results agree with the results from [7-11], although the comment in [10] about negative attitude towards technology among elderly users did not seem to apply to our user group. Our users also agreed with [12] on the importance of hands-free interaction. Added to this there were a number of detailed useful suggestions and observations.

If we consider the method used, we found that the sequence design used was very fruitful. The inclusion of existing technology in the focus group discussions provide a technological reference for the users, while the scenario walk provided the necessary context (during the walk comments were made that were not triggered in the preceding discussion). Concerning the diaries, the participants were concerned that the researchers should get as much as possible out of the diaries and suggested longer time for diary keeping. The workshop finally, showed that also for non-visual interaction design, low-fi workshops can be a useful tool for involving users in the design process (as expected from [13]). The workshop and discussion turned out to be a fruitful arena to get into detailed questions about the functionality of the system. During the simulated walk, both users and researchers were in the context of a way finding task (however artificial), which triggered questions and also made more plausible that both parties were talking about the same thing.

\section{Conclusion}

To conclude, we have shown that a more longitudinal study design consisting of a linked sequence combining a focus group discussion, a contextual test/interview, a diary study and a user workshop where users design low-fi versions of potential services can be a useful tool for the exploration of the user requirements for non-visual interaction designs intended for mobile navigation services. We note the importance of landmarks and information about objects in the environment. Other things seen to be important were hands-free and eyes-free operation, position accuracy, speech feedback, confirmation, routing design, correct user orientation in the environment and map content. Furthermore, we have seen that elderly sighted and visually impaired users appear to have many requirements in common in this study - although it should 
be noted that a request for hands-free and eyes-free solutions can also be a consequence of the mobile context (where hands and eyes are needed for other things).

\section{References}

1. Magnusson, C., Brewster, S., Sarjakoski, T., Roselier, S., Sarjakoski, L.T., Tollmar, K.: Exploring Future Challenges for Haptic, Audio and Visual Interfaces for Mobile Maps and Location Based Service. In: LocWeb 2009 Workshop, CHI 2009, Boston, MA, USA, April 4-April 9 (2009)

2. Dykes, J., MacEachren, A.M., Kraak, M.-J.: Exploring Geovisualization. In: Dykes, J., MacEachren, A.M., Kraak, M.-J. (eds.) Exploring geovisualization. Elsevier, Amsterdam (2005)

3. Svanæs, D., Seland, G.: Putting the Users Center Stage: Role Playing and Low-fi Prototyping Enable End Users to Design Mobile Systems. In: CHI 2004, Vienna, Austria (2004)

4. Sá, M., Carriço, L.: Low-Fi Prototyping for Mobile Devices. In: CHI 2006, Montréal, Canada, pp. 694-699. ACM, New York (2006)

5. Messeter, J., Brandt, E., Halse, J., Johansson, M.: Contextualizing mobile IT. In: Proceedings of the 5th Conference on Designing interactive Systems: Processes, Practices, Methods, and Techniques. DIS 2004, Cambridge, MA, USA, August 1-4. ACM, New York (2004)

6. Iacucci, G., Kuutti, K., Ranta, M.: On the move with a magic thing: role playing in concept design of mobile services and devices. In: Boyarski, D., Kellogg, W.A. (eds.) Proceedings of the 3rd Conference on Designing interactive Systems: Processes, Practices, Methods, and Techniques. DIS 2000, New York City, New York, United States, August 17-19. ACM, New York (2000)

7. May, A., Ross, T., Bayer, S., Tarkiainen, M.: Pedestrian navigation aids: information requirements and design implications. Personal Ubiquitous Comput. 7(6), 331-338 (2003)

8. Bradley, A., Dunlop, D.: An Experimental Investigation into Wayfinding Directions for Visually Impaired People. Personal Ubiquitous Comput. 9(6), 395-403 (2005)

9. Strothotte, T., Petrie, H., Johnson, V., Reichert, L.: MoBIC: user needs and preliminary design for a mobility aid blind and elderly travellers. In: Proceedings of the 2nd TIDE Congress, La Villette, Paris, April 26-28 (1995)

10. Goodman, J., Gray, P.: A Design Space for Location-Sensitive Aids for Older Users. In: $\mathrm{HCI}$ in Mobile Guides, Udine, Italy (2003)

11. Sjölinder, M.: Age-related decline and navigation in electronic environments, $\mathrm{PhD}$ thesis, Department of Psychology, Stockholm University (2006)

12. Heuten, W., Henze, N., Pielot, M., Boll, S.: Tactile Wayfinder: A Non-Visual Support System for Wayfinding. In: NordiCHI 2008, Lund, Sweden, October 20-22 (2008)

13. Magnusson, C., Brewster, S.: Proceedings of the workshop: Guidelines for Haptic Lo-Fi prototyping. NordiCHI 2008, Lund, Sweden (October 19 2008),

http: / /www.english. certec.lth.se/haptics /

Proceedings_10_fi_workshop.pdf (accessed 2009-01-26) 